\title{
Die Lungenfunktion: State-of-the-Art und neue Ergebnisse
}

\author{
W. T. Ulmer \\ T. Schäfer
}

The Lung Function: State-of-the-Art and New Results

Dieses Kolloquium wurde am 11.3.2003 in der Abteilung für Angewandte Physiologie (Leitung: Prof. Dr. M. E. Schläfke) der RuhrUniversität-Bochum abgehalten.

Das in drei Teile gegliederte Kolloquium, welches von SchultzeWerninghaus/Bochum, Rasche/Wuppertal und Wuthe/Berlin geleitet wurde, stand ganz unter der Aufgabe der Qualitätskontrolle und Qualitätsverbesserung in der Lungenfunktionsdiagnostik.

Für die Diagnostik, die Prognose, die Therapie und das Begutachtungswesen von Lungenerkrankungen sind Lungenfunktionsuntersuchungen oft unerlässlich. Mit 12 Referaten mit jeweils 15 Minuten Redezeit und ebensoviel Zeit für die Diskussionen wurden die wesentlichen Themen mit neuen Ergebnissen und dem Austausch von praktischen Erfahrungen abgehandelt.

Die angegebenen Indikationen wurden von Duchna/Bochum und Konietzko/Essen übereinstimmend hervorgehoben. Ein Verzicht würde für die Grundlagen und Qualität aller Indikationen große Unsicherheiten bedeuten.

Die älteste und am weitesten verbreitete Methodik ist die Spirometrie (Hutchinson 1844, 1846). Alle entsprechenden eingesetzten Parameter sind mit der Vitalkapazität verbunden, womit auch größere Unsicherheiten bestehen. Die von den Untersuchten und den Untersuchern angewandte „Vitalkapazitätsspirometrie“ ist von der Mitarbeit abhängig. Besonders die „end of test failure“ (Hankinson 1999) geben Anlass zu einem großen Prozentsatz unzureichender Ergebnisse. Die Ergebnisse werden dann meist auf die nach interindividuellen Messungen errechneten Sollwerte, die eine Streuung von \pm 20 (30)\% zeigen, bezogen. Die individuellen Sollwerte streuen aber mit $\pm 10 \%$ wesentlich weniger (Schäfer u. Mitarb. 2002). Hierin sind zwangsläufig Fehlinterpretationen begründet. Nur aus Langzeitergebnissen, die auf zuverlässige Erstbefunde zurückgreifen, sind repräsentative Aussagen möglich. Normalwerte von Gesunden können für $\mathrm{FEV}_{1}$ z.B. bis $140 \%$ reichen. Wenn ein derartiger Proband einen Befund von 100\% liefert, so liegt ein erhebliches Maß an Fehlinterpretation vor, wenn dies als Normalbefund bezeichnet wird. Einzelbefunde allein sind schwer oder nicht interpretationsfähig, auch wenn in vielen Empfehlungen der $\mathrm{FEV}_{1}$ als Maßstab gehandelt wird.

Zu den ganzkörperplethysmographischen Grundlagen (Ulmer/ Bochum) wurde hervorgehoben, dass es sich bei den zu gewinnenden Parametern, Strömungswiderstand in den Atemwegen und endexspiratorisches intrathorakales Gasvolumen, um mitarbeitsunabhängige Größen handelt. Mit der von der Fa. Jaeger/ Würzburg entwickelten elektrischen Stabilisation wird heutzutage eine hervorragende Reproduzierbarkeit der Ergebnisse erreicht (Schäfer u. Mitarb. 2002). In jedem Fall sollten immer 2 (3) Kurven aufgenommen werden, was einen minimalen zeitlichen wie auch methodischen Aufwand erfordert. Die Anschaffungskosten für das Gerät liegen allerdings etwas höher, was aber für die zu gewinnenden „Goldstandards“ auch in der Pneumologie zu akzeptieren ist. Die späteren Parameter „spezifische Resistance“ geben einmal den Versuch wieder, die Errechnung auf das intrathorakale Gasvolumen plus tatsächliches Atemzugvolumen und nicht auf das intrathorakale Gasvolumen (IGV) allein zu beziehen. Diese Korrekturen sind aber sehr gering. Von anderen Untersuchern wird die „spezifische Resistance“ als Rt/ IGV angegeben. Dies bringt aber keinerlei Gewinn, da alle Faktorenbildungen Informationsverlust bedeuten. Es ist wesentlich, die Strömungswiderstände und das IGV getrennt zu beachten, da beide Größen unabhängig voneinander verändert sein können, was unterschiedlichen Krankheitsprozessen entspricht. Um Fehlbestimmungen des IGV zu kompensieren, sollte kein Argument für eine derartige Berechnung sein, zumal bei der elektri- 
schen Kompensation solche Fehler nicht mehr akzeptiert werden können.

Herr Schäfer/Bochum berichtete über Korrelationen zwischen spirometrischen und ganzkörperplethysmographischen Parametern. Die Kenntnis solcher Beziehungen hilft sehr für die Zuordnung entsprechender Befunde. Die bei Spontanatmung erhobenen Resistancewerte (Rt) korrelieren signifikant mit den spirometrischen $\mathrm{FEV}_{1}$ - wie Vitalkapazitätsbefunden. Die gute Reproduzierbarkeit der IGV-Bestimmungen zeigt, dass bei gleicher Körpergröße, die für die Berechnung der Sollwerte einhergeht, unterschiedliche Lungengrößen vorhanden sind. $\mathrm{Zu}$ diesen Lungengrößen bestehen Korrelationen der Vitalkapazität, des $\mathrm{FEV}_{1}$ und der Rt. Größere Lungen haben entsprechend günstigere $\mathrm{Pa}$ rameter. In Zukunft ist zu entscheiden, ob die Lungengröße, unabhängig von der Körpergröße, in eine Sollwertformel einzubeziehen ist. Aber auch jetzt schon kann dies bei entsprechenden Konstellationen berücksichtigt werden.

Die neuen Aussagen, die „Resistance-Volumenanalysen“ ermöglichen, wurden von Schäfer und Duchna/Bochum dargestellt. Aus der Spontanatmung sind dabei wesentliche Parameter abzuleiten. Die Dynamik der Strömungswiderstände im Verlauf von Einzelatemzügen lässt sich zuverlässig erfassen. Bei welchen Volumina die Strömungswiderstände unter Umständen bis zum Verschluss der Atemwege ansteigen, wird dargestellt. Auch der Tonus des Bronchialsystems und die Veränderungen der Atemmechanik bei der Bronchodilatation sind zuverlässig erfassbar. Mit einer derartigen Analyse sind bei Spontanatmung ganz wesentliche Einblicke in das Atmungsgeschehen bei Gesunden und Patienten möglich. Die Korrelationen zu ganzkörperplethysmographischen Bestimmungen des Strömungswiderstandes bestätigen die Zuverlässigkeit dieses neuen, in der Praxis einfach zu handhabenden Verfahrens.

Die Oszilloresistometrie (Smith/Würzburg) stellt ein elegantes, von Atemzug zu Atemzug einsetzbares Verfahren zur Bestimmung der Strömungswiderstände in den Atemwegen dar. Gerade bei Provokationstesten leistet es wertvolle Hilfe, Veränderungen des Strömungswiderstandes sofort zu erfassen. Die Messungen bei verschiedenen Frequenzen der überlagerten Druckwellen geben die Möglichkeit, den Einfluss der größeren wie der peripheren Atemwege zu erfassen. Auch in der Pädiatrie liefert dieses Verfahren wertvolle mitarbeitsunabhängige Informationen.

Herr Klüpfel/Nürnberg berichtete über die Bedeutung des Transferfaktors als sensible und gut reproduzierbare Messgröße des Gasaustausches. Die Ergebnisse sind aber nicht spezifisch und erlauben nur generelle Aussagen über den Gasaustausch. Dem wurde auch Rechnung getragen, indem in der Literatur an Stelle der Bezeichnung „Diffusionsmessung“, der Terminus Bestimmung des „Transferfaktors“ eingeführt wurde.

Körperliche Belastung kann manchmal als zusätzlicher Test erforderlich sein, um an der „Grenze der Belastbarkeit“ zu prüfen, inwieweit dann auch der Gasaustausch betroffen ist (Wuthe/Berlin). Den Blutgasveränderungen, vorwiegend am arteriellen/kapillaren Sauerstoffdruck erkennbar, können Verteilungsstörungen zugrunde liegen. Deren Verhalten ist für die körperliche Belastung nicht vorauszusagen. Ein Absinken des Sauerstoffdru- ckes kann, wie meist, einer Verstärkung des Einflusses von Verteilungsstörungen wie auch einer Manifestation von Diffusionsstörungen entsprechen. Da sich Verteilungsstörungen auch bei durch körperliche Belastung gesteigerter Atmung verbessern können, werden auch Verbesserungen am arteriellen Sauerstoffdruck gemessen.

Über größere Erfahrungen am inhalativen unspezifischen Provokationstest trug Herr Hoffarth/Leverkusen vor. Der Mehrstufentest ist sehr sicher, aber im Verhältnis zum 1-Konzentrationstest deutlich zeitaufwendiger. Die Reproduzierbarkeit über längere Zeiträume zeigt, dass ein größerer Prozentsatz ( $50 \%)$ zu Normwerten zurückkehren kann. Auch für die Arbeitseinsatzlenkung sind solche Befunde eines „überempfindlichen Bronchialsystems“ zu berücksichtigen. Solche Probanden bedürfen einer kurzfristigeren Überwachung, auch mit klinischen Befunden.

Herr Rasche/Wuppertal stellte den Bronchodilatationstest zur Diskussion. Die Grenzangabe von $15 \%$ Verbesserung des $\mathrm{FEV}_{1}$ kann nicht als zuverlässig zur Einstufung von Respondern oder Nichtrespondern angesehen werden. Schon die alineare Beziehung von $\mathrm{FEV}_{1}$ und Rt zeigt, dass bei niedrigen $\mathrm{FEV}_{1}$-Werten größere Veränderungen von Rt eintreten können, die für die Patienten sehr bedeutsam sind. Für eine optimale Therapieeinstellung kann eine Testwiederholung mit höheren Dosen des Bronchodilatators und nach zusätzlicher Gabe von Glukokortikosteroiden erforderlich sein. Die Angabe von Patienten, dass es ihnen nach Inhalation eines Bronchodilatators besser geht, zeigt keinesfalls, dass dann auch die optimale Bronchodilatation sichergestellt ist. Die optimale Einstellung vermindert aber wesentlich auch die Rezidivhäufigkeit von Exazerbationen. Die konsequente optimale Bronchodilatation erlaubt dann erst, die irreversiblen Anteile einer IGV- oder/und Rt-Erhöhung zu erfassen.

Der Beitrag von Herrn Konietzko/Essen zur Lungenfunktionsdiagnostik in der Praxis zeigte dann, dass gerade in der heutigen Zeit Kompromisse über die Häufigkeit des Einsatzes der verschiedenen Methoden zu suchen und auch möglich sind. Das individuelle Krankheitsgeschehen geht hier in die Entscheidungen ein. Ein genereller Verzicht ist aber für alle Indikationsstellungen der Lungenfunktionsdiagnostik unverantwortlich und so nicht möglich. Die Qualität der Lungenfunktionsdiagnostik zeigt in der Praxis von der methodischen Durchführung bis zur Deutung der Ergebnisse große Differenzen, die auf besserem Niveau zu verkleinern als eine wichtige Aufgabe der Ausbildung von Ärzten angesehen werden muss.

\section{Literatur}

\footnotetext{
${ }^{1}$ Hutchinson J. Lecture on vital statistics, embracing an account of a new instrument for detecting the presence of disease of the system. Lancet 1844: 567-570, 594-596

${ }^{2}$ Hutchinson J. On capacity of lungs and on respiratory functions with view of establishing precise and easy method of detecting disease by spirometer. Trans med-chir Soc London 1846; 29: 137

${ }^{3}$ Hankinson JL. Does Poor Quality Render it Inpractical? Chest 1999; 116/2: 276-277

${ }^{4}$ Schäfer T, Happel A, Islamova S, Schläfke ME, Ulmer WT. Zur Stabilität lungenfunktionsanalytischer Parameter: Spirometrie, Bodyplethysmographie und Mundverschlußdruck (PO.1). Pneumologie 2002; 56: $679-683$
}

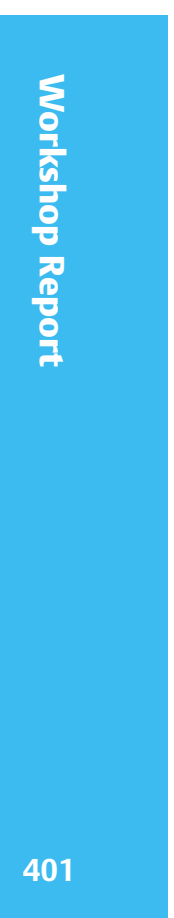

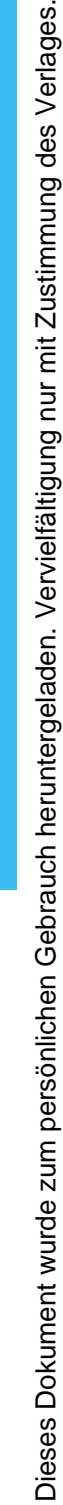

